\title{
Familie in der Stadt und am Land: Zur Bedeutung des regionalen Kontextes für die Realisierung des Kinderwunsches in Österreich
}

\author{
Bernhard Riederer • Isabella Buber-Ennser
}

Eingegangen: 27. August 2019 / Angenommen: 13. Juli 2020 / Online publiziert: 25. November 2020

(C) Der/die Autor(en) 2020, korrigierte Publikation 2020

Zusammenfassung Es ist seit Langem bekannt, dass sich Fertilitätsraten zwischen ländlichen und urbanen Regionen unterscheiden. Stadt-Land-Unterschiede bei der Realisierung bestehender Kinderwünsche wurden jedoch bisher kaum untersucht. Der vorliegende Aufsatz untersucht die Verwirklichung bestehender Kinderwünsche in Österreich, wobei ländliche und urbane Gebiete mit der Hauptstadt Wien als einziger Millionenstadt verglichen werden. Unsere Ergebnisse zeigen, dass Kinderwünsche in Wien seltener realisiert werden als in ländlichen oder anderen urbanen Regionen Österreichs. Unterschiede in der Bevölkerungszusammensetzung erklären das häufigere Aufschieben des Kinderwunsches in Wien. Für die Aufgabe des DreiJahres-Kinderwunsches bestehen aber auch nach Berücksichtigung sozioökonomischer und demographischer Charakteristika sowie Wertvorstellungen noch regionale Unterschiede. Dies könnte an der Vielfalt an bildungs- und berufsbezogenen Optionen in Städten liegen, die mit einer Familiengründung konkurrieren.

Schlüsselwörter Kinderwunsch · Fertilität · Stadt-Land-Unterschiede · Generations and Gender Survey (GGS) · Wien

\footnotetext{
B. Riederer $(\bowtie)$

Institut für Soziologie, Universität Wien, Rooseveltplatz 2, 1090 Wien, Österreich

E-Mail: bernhard.riederer@univie.ac.at

B. Riederer · I. Buber-Ennser

Institut für Demographie/ÖAW, Wittgenstein Centre (IIASA, ÖAW, Universität Wien), Vordere

Zollamtsstraße 3, 1030 Wien, Österreich

E-Mail: isabella.buber-ennser@oeaw.ac.at
} 


\title{
Family in urban and rural regions: The relevance of regional context for the realization of childbearing intentions in Austria
}

\begin{abstract}
It is well known that fertility rates differ between rural and urban regions. Nevertheless, regional differences in realization of childbearing intentions have been hardly studied. The present article analyses the realization of short-time childbearing intentions in Austria, comparing rural areas, urban areas, and Vienna (the capital is the only city with more than 1 million inhabitants in Austria). Our findings demonstrate that realization is lower in Vienna than in other urban or rural regions of Austria. Different population compositions contribute to a more frequent postponement of intentions in Vienna. Regional differences in abandonment of fertility intentions remain after controlling for socio-economic characteristics, demographics, and attitudes. The higher number of available options regarding education, employment, and lifestyles in cities might compete with family formation and increase the costs of childbearing.
\end{abstract}

Keywords Childbearing intentions · Fertility · Urban-rural differences · Generations and Gender Survey (GGS) · Vienna

\section{Einleitung}

Der Unterschied zwischen Stadt und Land ist ein in der deutschsprachigen Soziologie von Beginn an relevantes Thema. Dabei wird durchaus auch Bezug auf die Familie genommen. Laut Tönnies (1887) etwa kennzeichnet die ländlichen Dörfer die Gemeinschaft, während in der Stadt Gesellschaft vorherrscht. Gerade für das ländliche Zusammenleben sind familiäre Bindungen dabei von großer Bedeutung. In der Stadt aber sind die Beziehungen zwischen den Menschen loser und die Menschen agieren rationaler (Simmel 1995 [1903]). Indem sie den Menschen aus traditionellen Bindungen löst, ermöglicht die Stadt aber auch mehr Entscheidungsfreiheit. Eine dieser Entscheidungen ist die Entscheidung für ein Kind, deren Realisierung im Mittelpunkt der vorliegenden Arbeit steht.

Dass das städtische Leben die Entwicklung der Familie beeinflusst, demonstrieren die Auswirkungen der zunehmenden Urbanisierung auf den demographischen Wandel (Jaffe 1942; Bocquier und Costa 2015; Sharlin 1986). Aber auch der Geburtenrückgang nach dem Babyboom der 1960er-Jahre vollzog sich zunächst in der Stadt, Hand in Hand mit dem Anstieg des Bildungsniveaus und der Berufstätigkeit der Frauen (z.B. Galloway et al. 1998). So weisen Städte in der Regel bis heute geringere Fertilitätsraten als ländliche Regionen auf (Hank 2002; Kulu und Washbrook 2014). Innerhalb Österreichs kennzeichnen städtische Gemeinden u.a. eine etwas geringere Fertilitätsrate (1,40 versus 1,47$)$, ein höheres durchschnittliches Alter bei der Geburt des ersten Kindes $(28,3$ versus 27,9) und eine geringere Unehelichenquote (35,5 versus 43,5\%) als ländliche Gemeinden (Daten beziehen sich auf den Durchschnitt 2002-2017; Wisbauer und Klotz 2019). Studien, die sich mit dem Kinderwunsch und dessen Umsetzung beschäftigen, berücksichtigten bislang aber 
kaum den regionalen Kontext. Der vorliegende Artikel soll diese Forschungslücke ein Stück weit schließen helfen.

Mit unserer Studie tragen wir zur Forschung zur Realisierung von Kinderwünschen bei, indem wir explizit zwischen einer Metropole (dem Großraum Wien), urbanen Regionen und ländlichen Regionen unterscheiden. Zudem lohnt es sich aus zweierlei Gründen, die Situation in Österreich näher zu betrachten. Erstens bezieht sich ein Großteil der existierenden Studien zu Stadt-Land-Unterschieden in der Fertilität auf die nordischen Staaten (Kulu und Washbrook 2014). Zweitens wurde die Realisierung von Kinderwünschen in Österreich wegen fehlender Paneldaten seit Anfang der 1980er-Jahre nicht mehr untersucht (Gisser et al. 1985). Damals wurden allerdings nur junge verheiratete Frauen befragt (Frauen, die 1978 weniger als fünf Jahre verheiratet und zum Zeitpunkt der Eheschließung unter 30 waren). Dabei war ein deutliches Stadt-Land-Gefälle ersichtlich: Frauen in ländlichen Gebieten erfüllten ihre Kinderwünsche eher als Frauen in Wien. Im multiplen Regressionsmodell war der Stadt-Land-Unterschied jedoch deutlich geringer. Die Autoren führten das auf Unterschiede in der Zusammensetzung von Stadt- und Landbevölkerung zurück (v. a. bez. des Berufs des Mannes und der Erwerbsorientierung der Frau).

Die vorliegende Arbeit basiert auf dem „Generations and Gender Survey (GGS)“. Dabei wurden Frauen und Männer im Alter von 18 bis 45 Jahren 2008/2009 ausführlich zu Partnerschaft und Familie befragt, auch zum Kinderwunsch für die kommenden drei Jahre sowie zum generellen Wunsch, (weitere) Kinder zu bekommen. Dieselben Personen wurden 2012/2013 wieder kontaktiert. Dieser Beitrag fokussiert auf die Realisierung des 2008/2009 geäußerten Wunsches, innerhalb der nächsten drei Jahre ein Kind zu bekommen.

\section{Realisierung des Kinderwunsches und regionaler Kontext}

Die bisherige Forschung hat gezeigt, dass eine Vielzahl individueller Faktoren (z. B. Alter, Partnerschaft, Anzahl der Kinder (Parität), Bildung) die Wahrscheinlichkeit der Erfüllung des Kinderwunsches beeinflusst (z. B. Kapitány und Spéder 2012; Régnier-Loilier und Vignoli 2011). Aller bestehenden Unterschiede in der Fertilität zum Trotz wurde der regionale Kontext dabei aber kaum berücksichtigt. Selbst Studien, die regionale Unterschiede ins Auge fassten (Mencarini et al. 2015; Rinesi et al. 2011; Kuhnt und Trappe 2016), enthielten keine systematischen StadtLand-Vergleiche oder befassten sich nicht explizit mit Großstädten. So unterschieden Mencarini et al. (2015) zwischen großen, mittleren und kleinen Gemeinden. Riederer und Buber-Ennser (2019) fokussierten zwar auf Stadt-Land-Unterschiede, analysierten jedoch zum einen Daten von elf europäischen Ländern gemeinsam und differenzierten zum anderen nicht zwischen größeren und kleineren Städten.

Während biologische Ansätze zur Umsetzung des Kinderwunsches primär auf die Fruchtbarkeit eingehen, thematisieren makrosoziologische Theorien und die Thesen des demographischen Übergangs (van de Kaa 1987; Lesthaeghe 1995) die wirtschaftliche Entwicklung, verbesserte Lebensbedingungen und den Wertewandel (Individualisierung, Emanzipation etc.), die sich auf den Wunsch, (viele) Kinder zu bekommen, auswirken (vgl. Neuwirth et al. 2011). Theorien zu Motiven, Kinder 
zu bekommen (z. B. Value of Children, siehe Nauck 2001), oder zur Entstehung des Kinderwunsches (z. B. Theory of Planned Behaviour, siehe Ajzen und Klobas 2013), behandeln nur eingeschränkt regionale Aspekte. ${ }^{1}$ Sie konzentrieren sich auf Unterschiede zwischen Nationalstaaten, besprechen Einflüsse auf den Kinderwunsch, nicht aber auf dessen Realisierung, oder erwähnen situative Einflüsse und Umweltfaktoren, ohne deren genaue Wirkung zu spezifizieren. Ansatzpunkte zur Rolle des regionalen Umfelds für die Umsetzung des Kinderwunsches bieten eher Theorien zu Stadt-Land-Unterschieden in der Fertilität.

Eine frühe Erklärung für eine geringere Fertilität in Städten kann man bei Michael T. Sadler (1780-1835) oder Herbert Spencer (1820-1903) finden, die eine größere Bevölkerungsdichte mit geringerer Fruchtbarkeit assoziierten (vgl. Ferdinand 1999, S. 104; Neuwirth et al. 2011, S. 14). In der Literatur werden aktuell verschiedene Erklärungen für Fertilitätsunterschiede zwischen urbanen und ländlichen Regionen angeführt, die vor allem regionale Opportunitätsstrukturen, soziale Normen sowie die unterschiedliche Bevölkerungszusammensetzung als zentral betrachten (Hank 2002; Trovato und Grindstaff 1980). Letztere ist allerdings häufig nicht von den ersten beiden zu trennen, da sich auch die Bevölkerungszusammensetzung zum Teil aus den Opportunitäten und durch Gruppen mit unterschiedlichen Werthaltungen und Einstellungen ergibt. ${ }^{2}$ Hinzu kommt unserer Ansicht nach jedoch ein weiterer Aspekt, der für die Realisierung bestehender Kinderwünsche hochrelevant sein könnte: Wenn im Zuge steigender Anforderungen an Eltern die persönliche Frage, ob man für Kinder bereit ist, zum Thema wird, gewinnt auch die Frage nach einem für Kinder geeigneten Wohnumfeld an Bedeutung (Buber-Ennser und Fliegenschnee 2013; Riederer 2018).

Opportunitäten werden in verschiedensten sozioökonomischen und soziologischen Theorien zum Geburtenrückgang erwähnt, die diesen mit veränderten Rahmenbedingungen und konkurrierenden (biographischen) Alternativen erklären (vgl. Neuwirth et al. 2011). Sie spielen z.B. in der bekannten ,biographischen Fertilitätstheorie“" von Herwig Birg eine große Rolle (Birg et al. 1991). Entscheidungen mit langfristigen Folgen, wie die Geburt eines Kindes, führen zu einer Einschränkung der weiteren Optionen (und daher zu biographischen Opportunitätskosten). Ein Aufschub des Kinderwunsches oder gar dessen Aufgabe können die Folge sein. Variierende Opportunitätskosten können zudem auch regionale Unterschiede erklären (Birg und Flöthmann 1990).

Gerade bildungs- und berufsspezifische Möglichkeiten sind relevant für die Realisierung des Kinderwunsches, da sie zu einem Aufschub der Realisierung oder wo-

\footnotetext{
1 Diese Ansätze können im handlungstheoretischen Paradigma verortet werden. Die Handlung ist streng genommen allerdings der Sexualakt (das Handeln) bzw. der Verzicht auf Verhütungsmethoden (das Nicht-Handeln) und nicht die Geburt eines Kindes. In unserem Aufsatz geht es jedoch nicht um die Frage der (rationalen oder nicht-rationalen) Entscheidung für ein Kind, sondern um die Umsetzung oder Nicht-Umsetzung eines einmal bestehenden Kinderwunsches. Die Handlungstheorie könnte hier nur beitragen, wenn man davon ausgeht, dass der Kinderwunsch alleine deshalb nicht realisiert wird, weil er sich geändert hat (sich eine Person nun anders entschieden hat).

2 So führen andere Bildungsmöglichkeiten in der Stadt (Opportunitäten) zu größeren Anteilen an Höhergebildeten und sind traditionellere Einstellungen am Land (Werte/Normen) möglicherweise auf einen geringeren Anteil an Konfessionslosen zurückzuführen.
} 
möglich zu einer Aufgabe des ursprünglichen Kinderwunsches führen können. Städte bieten in der Regel mehr bildungs- und berufsspezifische Möglichkeiten als ländliche Regionen. Das gilt innerhalb Österreichs insbesondere für Wien. Die Hauptstadt kennzeichnen im Vergleich zu Österreich insgesamt deutlich höhere Anteile an Studierenden sowie Personen mit tertiärem Bildungsabschluss oder Beschäftigung im Wissenschafts- und Technologiesektor wie auch ein größerer Dienstleistungssektor (Statistik Austria 2019a).

Die Realisierung von Kinderwünschen betreffend werden Opportunitätsstrukturen zudem stark von der Familienpolitik mitbestimmt. Österreichs Familienpolitik charakterisierte lange Zeit das hohe Niveau an finanzieller Unterstützung für Familien (Ferrarini 2006; Korpi 2000). In den letzten Jahrzehnten wurden zusätzlich Maßnahmen zur Verbesserung der Vereinbarkeit von Familie und Beruf eingeführt. Diese betrafen vor allem den Ausbau der Kinderbetreuung für Vorschulkinder (Blum et al. 2014). Gerade bei der Betreuung der Jüngsten (unter 3 Jahre) unterscheidet sich Wien jedoch von anderen Regionen Österreichs und weist deutlich höhere Betreuungsquoten auf (Statistik Austria 2019b). Verfügbare Kinderbetreuung sollte Opportunitätskosten senken, dadurch die Umsetzung des Kinderwunsches erleichtern und dem aufschiebenden Effekt größerer beruflicher Möglichkeiten entgegenwirken.

Was Normen und Werte betrifft, ist zunächst anzumerken, dass in Österreich wie in den meisten europäischen Ländern - das Ideal der Zwei-Kind-Familie dominiert (Sobotka und Beaujouan 2014; Riederer 2005). Für den Großteil der Menschen in Österreich gehören Kinder zu einer gelungenen Partnerschaft (Berghammer und Schmidt 2019) und einem geglückten Leben (Berghammer et al. 2019). Trotz einer generellen Annäherung in den letzten drei Jahrzehnten bestehen zudem weiterhin in ländlichen Regionen und Kleinstädten traditionellere Einstellungen als in größeren Städten (Beham-Rabanser et al. 2019). Auch die Meinung, dass Kinder notwendig für ein erfülltes Leben sind, wird in ländlichen Regionen öfter vertreten als in städtischen (Riederer und Buber-Ennser 2019). Da in ländlichen Regionen traditionellere Ansichten zur Familie häufiger sind, könnte die Elternschaft als Teil der Lebensplanung für Personen im ländlichen Raum wichtiger sein. Dies könnte zu einer höheren Realisierung von Kinderwünschen am Land führen.

$\mathrm{Zu}$ Unterschieden zwischen Stadt und Land könnten auch Unterschiede in der Religiosität der Bevölkerung beitragen. Obwohl in Österreich - wie in anderen westeuropäischen Ländern - die Religiosität abnimmt, ist Religion ein relevanter Faktor im Geburtsverhalten und bei der Gestaltung von Lebensverläufen (Goujon et al. 2007; Berghammer 2012). Ideale und gewünschte Kinderzahl sind unter religiösen Menschen höher als bei Personen, die keiner Glaubensgemeinschaft angehören (Philipov und Berghammer 2007). Die Daten des Generations and Gender Surveys zeigen eindeutige Unterschiede in der Teilnahme an religiösen Zusammenkünften: Während nur rund 17 bis $20 \%$ der 18-45-Jährigen im Großraum Wien oder anderen urbanen Regionen monatlich oder öfter Gottesdienste, das Freitagsgebet in einer Moschee o. Ä. besuchen, sind es im ländlichen Raum $30 \%$ (eigene Berechnungen). Schließlich gibt es markante Unterschiede nach Religionszugehörigkeit, mit höheren Kinderzahlen unter Frauen muslimischen Glaubens und niedrigeren Kinderzahlen unter konfessionslosen Frauen (Prskawetz et al. 2008; Sobotka und Zeman 2015). 
In Wien ist jedoch nicht nur der Anteil der muslimischen Frauen höher als im Rest des Landes, sondern auch jener der konfessionslosen (Goujon et al. 2017).

Die österreichische Gesellschaft ist durch Zuwanderung geprägt. Aktuell sind rund $20 \%$ der in Österreich lebenden Menschen im Ausland geboren (Riederer et al. 2020). Der Anteil an Immigrantinnen ist in urbanen Zentren wesentlich höher als im ländlichen Raum (Wisbauer und Klotz 2019). Neben Gruppen mit höherer Fertilität (z. B. Frauen aus Syrien oder der Türkei) finden sich jedoch auch solche mit geringer Fertilität (z. B. Frauen aus Deutschland oder Ungarn) (Zeman et al. 2019). Ein höherer Anteil an ImmigrantInnen muss deshalb nicht zwingend zu höherer Realisierung beitragen. Gerade in Wien ist die migrantische Bevölkerung sowohl in Bezug auf die Herkunft als auch die berufliche Positionierung sehr heterogen $(24 \%$ in Führungspositionen oder akademischen Berufen, aber auch 19\% HilfsarbeiterInnen) (Riederer et al. 2019, S. 3).

Ein kindgerechtes Umfeld wird als wesentliche Voraussetzung für die ,,verantwortete Elternschaft“ (Kaufmann 1990) angesehen. In diesem Zusammenhang wird die Wohnsituation - insbesondere Wohneigentum und Wohnfläche - mit Familiengründung und Familienerweiterung assoziiert (Vignoli et al. 2013; Mulder 2006). Wien kennzeichnet innerhalb Österreichs ein besonders hoher Anteil an Mietwohnungen. Wohneigentum korreliert in Österreich stark mit der Gemeindegröße, und die durchschnittliche Wohnfläche pro Person ist im Hauseigentum größer als im Wohnungseigentum oder in Gemeindewohnungen. Hinzu kommt, dass Mietpreise in der Stadt in der Regel höher sind als am Land (Statistik Austria 2018). Eine damit verbundene Frage ist, ob man sich Kinder leisten kann. Es zeigt sich, dass der Anteil an Menschen, die finanzielle Probleme angeben, im Großraum Wien höher ist als in den übrigen Teilen des Landes. Die Nachbarschaft und die Umweltqualität sind weitere wichtige Faktoren eines kindgerechten Umfelds. Größere Gemeinden kennzeichnen eine höhere Belastung durch Lärm, Luft- und Umweltverschmutzung sowie ein häufigeres Auftreten von Kriminalität und Vandalismus (Statistik Austria 2018). Ländliche Regionen könnten daher häufiger als kindgerecht ${ }^{3}$ angesehen werden.

Aufgrund der oben beschriebenen Zusammenhänge und Unterschiede nehmen wir an, dass bestehende Kinderwünsche in Städten - und im Besonderen in Wien - weniger oft realisiert werden als in ländlichen Gebieten. Abgesehen von der Verfügbarkeit von Kinderbetreuungseinrichtungen sprechen sämtliche besprochenen Faktoren (u. a. Werte und Normen) für diese Hypothese. Städte bieten ihren BewohnerInnen mehr Möglichkeiten, die mit einer Familiengründung konkurrieren (v. a. bildungsund berufsbezogene Optionen), und ein mancherorts für Kinder weniger geeignetes Umfeld (z. B. Wohnqualität, Sicherheit). In Österreich könnte sich das vor allem im Vergleich der Metropole Wien mit ländlichen Gebieten zeigen.

\footnotetext{
3 Unter einem „,kindgerechten Umfeld“ kann Verschiedenes verstanden werden. Wir folgen hier dem OECD-Kinderbericht, demzufolge v. a. das materielle Wohlergehen, die Wohn- und Umweltbedingungen, Bildung, Gesundheit und Sicherheit, das Risikoverhalten und die Qualität des schulischen Umfelds relevant sind (OECD 2009). Eine ähnliche Definition, die u. a. Umweltbedingungen und Gefährdungen beinhaltet, findet sich auch im nationalen Aktionsplan für die Rechte von Kindern und Jugendlichen (BMSGK 2004, S. 60).
} 


\section{Daten, Variablen und Analysestrategie}

Die vorliegende Arbeit verwendet Paneldaten des „Generations and Gender Surveys (GGS)“ mit detaillierten Angaben zu Familiengründung und Fertilität (Vikat et al. 2007). In Österreich wurden bisher zwei Befragungswellen durchgeführt, nämlich 2008/2009 und 2012/2013. Die Erstbefragung ist repräsentativ für die österreichische Bevölkerung zwischen 18 und 45 Jahren in Bezug auf Alter, Geschlecht, Erwerbsstatus, Geburtsland, Lebensformen und Kinderzahl (Buber 2010). In der Wiederbefragung nahmen knapp acht von zehn Befragten wieder teil. Die zweite Welle ist in Bezug auf traditionelle Einstellungen, Bildung und Geburtsland leicht verzerrt, eine markante Selektivität ist aber nicht gegeben (Buber-Ennser 2014).

Unterschiede in der regionalen Fertilität können aus drei Gründen zustande kommen: a) weil Personen mit Kinderwunsch am Land stärker vertreten sind als in der Stadt, b) weil Personen mit Kinderwunsch diesen in ländlichen Regionen häufiger realisieren als im städtischen Umfeld, oder c) weil ein Teil der StadtbewohnerInnen mit starkem Kinderwunsch und/oder hohem Umsetzungswillen in ländliche Regionen umzieht, weil sie diese als kindgerechter ansehen. Um Möglichkeit a) nachzugehen, betrachten wir zunächst den Kinderwunsch zum Zeitpunkt der ersten Erhebung. Möglichkeit b) steht danach im Fokus der vorliegenden Studie. Eine aussagekräftige Analyse zur Möglichkeit c) war hingegen aufgrund der geringen Fallzahl der Wohnortwechsel im Sample nicht möglich und bedarf einer Panel-Befragung über einen längeren Zeitraum.

Der Kinderwunsch ist unsere Ausgangsvariable und basiert auf drei Fragen, die in beiden Erhebungswellen gestellt wurden, nämlich 1) „Möchten Sie selbst jetzt ein (weiteres) Kind?“ (ja, nein, weiß nicht), 2) „Haben Sie vor, in den nächsten drei Jahren ein Kind zu bekommen?" (ganz sicher nicht, wahrscheinlich nicht, wahrscheinlich ja, ganz sicher ja) und 3) „Nehmen wir einmal an, Sie würden in den nächsten drei Jahren kein Kind bekommen, möchten Sie denn überhaupt ein Kind bzw. noch weitere Kinder?" (ganz sicher nicht, wahrscheinlich nicht, wahrscheinlich ja, ganz sicher ja). Daraus generierten wir die Variable „Kinderwunsch“ mit folgenden Ausprägungen: 1) ein Kind innerhalb der nächsten drei Jahre, 2) später Kinder und 3) keine Kinder (mehr). ${ }^{4}$ Es sei erwähnt, dass die oben genannten Fragen nicht an Frauen und Männer gestellt wurden, die zum Zeitpunkt der Befragung ein Kind erwarteten. Auch Personen in gleichgeschlechtlichen Partnerschaften, Männer mit Partnerinnen über 50 Jahren und Personen, die angaben, unfruchtbar zu sein, wurden nicht zu ihren Kinderwünschen befragt. Aufgrund geringer Fallzahlen und großer Unsicherheit beim Kinderwunsch unter den Personen im Alter von 18 bis 20 Jahren, fokussieren wir auf Männer und Frauen im Alter zwischen 21 und 45.

Für die Realisierung des Kinderwunsches wurde das Geburtsverhalten zwischen Erst- und Zweitbefragung betrachtet. Dabei beschränken wir uns auf jene, die in der ersten Welle angaben, sich innerhalb der nächsten drei Jahre ein Kind zu wünschen.

\footnotetext{
${ }^{4}$ In Österreich waren die Interviewerinnen und Interviewer angewiesen, wenn möglich „Weiß nicht“-Antworten zu vermeiden und zu erfragen, ob es sich bei unsicherem Kinderwunsch eher um ein ,wahrscheinlich ja“ oder um ein ,wahrscheinlich nein“" handelte. Wenige Personen, die alle Kinderwunsch-Fragen mit „weiß nicht“ beantworteten, wurden in der vorliegenden Analyse ausgeschlossen.
} 
Bekamen Personen bis zur Zweitbefragung ein Kind oder erwarteten sie eines, so sprechen wir von ,Realisierung des Kinderwunsches““.5 Waren sie zwischenzeitlich nicht Eltern eines Neugeborenen geworden, so unterscheiden wir weiter - in Anlehnung an frühere Studien (Spéder und Kapitány 2009; Kapitány und Spéder 2012) in Aufschieben und Aufgeben des Kinderwunsches, falls in der Zweitbefragung ein oder kein weiterer Kinderwunsch geäußert wurde.

In der vorliegenden Studie ist der regionale Kontext der wichtigste erklärende Faktor, wobei wir zwischen 1) dem Großraum Wien, 2) urbanen Regionen und 3) ländlichen Regionen unterscheiden. Mit dem Begriff „Großraum Wien“ bezeichnen wir Wien und das Wiener Umland. Letzteres beinhaltet im Norden die Bezirke Korneuburg, Tulln und Teile der Bezirke Gänserndorf, Mistelbach und Wien-Umgebung und im Süden die Bezirke Bruck an der Leitha, Baden und Mödling sowie Teile des Bezirks Wien-Umgebung (Statistik Austria 2015). Die Unterscheidung in urbane und ländliche Regionen beruht auf der regionalen Typologie der OECD (2011), die zwischen Regionen mit hoher, mittlerer und niedriger Bevölkerungsdichte unterscheidet. Als urbane Regionen bezeichnen wir Orte mit hoher oder mittlerer Bevölkerungsdichte außerhalb des Großraums Wien (z.B. Linz, Graz, Salzburg, Innsbruck). Ländliche Regionen sind Orte mit niedriger Bevölkerungsdichte (weniger als 150 Einwohner pro $\mathrm{km}^{2}$ ). Diese Dreiteilung dichotomisieren wir für manche Analysen (,Großraum Wien“ versus ,,andere Regionen“).

Verschiedene sozio-demographische und ökonomische Charakteristika haben sich als bedeutend für die Realisierung von Kinderwünschen erwiesen (Spéder und Kapitány 2009, 2014; Régnier-Loilier und Vignoli 2011). Folgende Größen werden als Kovariate in unsere Analysen einbezogen, wobei sich die Angaben auf die Welle 1 beziehen: a) Geschlecht; b) Alter (21-24, 25-34, 35-45 Jahre); c) Partnerschaftsstatus (verheiratet, unverheiratet zusammenlebend, ,living apart together“ (LAT), partnerlos); d) Elternschaft (kinderlos, 1 Kind, 2 Kinder, 3 oder mehr Kinder); e) höchster Bildungsabschluss (Primär- oder Sekundärbildung, tertiäre Bildung); f) ökonomische Lage (Situation (sehr) schwierig, Situation ok, Situation (sehr) gut). Zudem berücksichtigen wir: g) Einstellung zur Elternschaft (Kind(er) notwendig für ein erfülltes Leben, weder/noch, Kind(er) nicht notwendig für ein erfülltes Leben); h) Geburtsland (Österreich, anderes Land); i) die Beeinflussung der Fertilitätsentscheidung durch die Wohnverhältnisse (keineswegs, etwas, stark); ${ }^{6} \mathrm{j}$ ) Religiosität, gemessen an der Häufigkeit des Kirchgangs oder der Teilnahme am Freitagsgebet in der Moschee o. Ä. ${ }^{7}$ (nie, weniger als einmal im Monat, einmal im Monat oder öfter).

\footnotetext{
5 Aus budgetären Gründen fand in Österreich die Zweitbefragung nach rund vier Jahren statt. In unserem Analysesample lagen zwischen Erst- und Zweitbefragung durchschnittlich 48 Monate. Sensitivitätsanalysen zeigen, dass eine Einschränkung auf Geburten innerhalb von drei Jahren zu sehr ähnlichen Ergebnissen führt.

6 „Wie stark würde Ihre Entscheidung, in den nächsten drei Jahren (doch) ein (weiteres) Kind zu bekommen von der Wohnsituation abhängen?": Antwortskala: (1) überhaupt nicht, (2) ein bisschen, (3) ziemlich stark, (4) sehr stark.

${ }^{7}$ Basierend auf der Frage: „Wie oft nehmen Sie an Gottesdiensten (bzw. religiösen Zusammenkünften) teil; abgesehen von Hochzeiten, Begräbnissen, Taufen und ähnlichem?“”.
} 
Tab. 1 Charakteristika des Analysesamples nach Region

\begin{tabular}{|c|c|c|c|}
\hline Verteilung in \% & $\begin{array}{l}\text { Großraum } \\
\text { Wien }\end{array}$ & $\begin{array}{l}\text { Urbane } \\
\text { Regionen }\end{array}$ & $\begin{array}{l}\text { Ländliche } \\
\text { Regionen }\end{array}$ \\
\hline \multicolumn{4}{|l|}{ Geschlecht } \\
\hline Männlich & 46 & 41 & 47 \\
\hline Weiblich & 54 & 59 & 53 \\
\hline \multicolumn{4}{|l|}{ Alter } \\
\hline 21-24 Jahre & 10 & 11 & 9 \\
\hline 25-34 Jahre & 54 & 62 & 62 \\
\hline 35-45 Jahre & 36 & 27 & 29 \\
\hline \multicolumn{4}{|l|}{ Partnerschaftsstatus } \\
\hline Verheiratet & 34 & 36 & 36 \\
\hline Unverheiratet zusammenlebend & 30 & 30 & 31 \\
\hline LAT & 18 & 18 & 19 \\
\hline Kein/e Partner/in & 19 & 16 & 14 \\
\hline \multicolumn{4}{|l|}{ Elternschaft (Parität) } \\
\hline Kinderlos & 61 & 56 & 55 \\
\hline 1 Kind & 30 & 26 & 27 \\
\hline 2 Kinder & 6 & 13 & 13 \\
\hline 3 oder mehr Kinder & 3 & 6 & 6 \\
\hline \multicolumn{4}{|l|}{ Höchster Bildungsabschluss } \\
\hline Primär- oder Sekundärbildung & 69 & 72 & 83 \\
\hline Tertiärer Bildungsabschluss & 26 & 24 & 14 \\
\hline Unbekannt & 4 & 4 & 3 \\
\hline \multicolumn{4}{|l|}{ Ökonomische Lage } \\
\hline Situation (sehr) schwierig & 29 & 25 & 16 \\
\hline Situation ok & 31 & 32 & 38 \\
\hline Situation (sehr) gut & 40 & 43 & 46 \\
\hline \multicolumn{4}{|l|}{ Einstellung zur Elternschaft } \\
\hline $\begin{array}{l}\text { Kind(er) notwendig für ein erfülltes } \\
\text { Leben }\end{array}$ & 22 & 27 & 32 \\
\hline Weder/noch & 23 & 27 & 24 \\
\hline Kind(er) nicht notwendig & 54 & 46 & 44 \\
\hline \multicolumn{4}{|l|}{ Geburtsland } \\
\hline Österreich & 70 & 81 & 91 \\
\hline Anderes Land & 30 & 19 & 9 \\
\hline \multicolumn{4}{|c|}{ Wohnverhältnisse beeinflussen Entscheidung für Kind } \\
\hline ... keineswegs & 37 & 50 & 55 \\
\hline ... ein bisschen & 35 & 23 & 23 \\
\hline ... stark & 28 & 28 & 23 \\
\hline \multicolumn{4}{|l|}{ Häufigkeit des Kirchgangs (o.̈̈.) } \\
\hline $\mathrm{Nie}$ & 56 & 42 & 24 \\
\hline Weniger als einmal im Monat & 29 & 31 & 40 \\
\hline Einmal im Monat oder öfter & 15 & 27 & 36 \\
\hline$N$ & 236 & 344 & 363 \\
\hline
\end{tabular}

Quelle: GGS Welle 1 und 2; Panelbefragte, die zum Zeitpunkt der ersten Erhebung binnen drei Jahre ein Kind haben wollten 
Eine Berücksichtigung dieser Kovariate ist aus zumindest zwei Gründen relevant. Neben der Bedeutung, die diese persönlichen Merkmale für die Umsetzung des Kinderwunsches haben, ist vor allem wesentlich, dass die unterschiedliche Zusammensetzung der regionalen Bevölkerungen nach diesen Merkmalen entscheidend zu Stadt-Land-Unterschieden in der Realisierung beitragen dürfte. Unterschiede in diesen Variablen - z. B. in der Religiosität, dem Kindern zugeordneten Stellenwert, dem Partnerschaftsstatus, der Bildung oder dem Alter der Bevölkerungen mit Kinderwunsch - sollten daher Stadt-Land-Unterschiede in der Realisierung des Kinderwunsches zumindest miterklären können. Solche Kompositionseffekte ergeben sich etwa dann, wenn die Landbevölkerung religiöser ist und die Notwendigkeit der Elternschaft für ein geglücktes Leben stärker betont, und es deshalb zu höherer Realisierung in ländlichen Regionen als in urbanen Regionen kommt. Auch ein höherer Anteil an partnerlosen Personen in der Stadt könnte dazu beitragen, dass die Realisierung geringer ist als am Land. Tatsächlich nehmen in unserem Analysesample (Tab. 1) Menschen aus ländlichen Regionen regelmäßiger an religiösen Zeremonien teil. Sie sehen Kinder auch häufiger als Notwendigkeit an. Der Anteil der Partnerlosen an den Personen, die sich ein Kind wünschen, ist hingegen in Wien am höchsten. Zum Aufschub des Kinderwunsches dürfte zudem beitragen, dass in Wien und anderen urbanen Regionen der Anteil an Personen mit tertiärer Bildung höher ist als in ländlichen Regionen. Zu einer häufigeren Aufgabe des Kinderwunsches in Wien könnte das im Vergleich zu den anderen Regionen höhere Alter der Personen mit Kinderwunsch führen.

Zweitens dürfte es zu interessanten Stadt-Land-Unterschieden bei den Kovariaten kommen. Es ist durchaus plausibel, dass die Wohnsituation vor allem in urbanen Regionen eine Rolle bei der Umsetzung vorhandener Kinderwünsche spielt. Auch die Bildung könnte in der Stadt, die höher Gebildeten andere Optionen bietet als ländliche Regionen, von größerer Bedeutung sein. Allerdings sind nicht nur die Opportunitätskosten in der Stadt höher. Bessere Kinderbetreuungsmöglichkeiten erlauben möglicherweise erst die Vereinbarkeit von Familie und Beruf. Man könnte auch so weit gehen, zu spekulieren, dass das Alter, die Ehe und die Elternschaft am Land von größerer Relevanz für die Realisierung sind als in der Stadt, wo die späte Elternschaft, unverheiratet zusammenlebende Paare und kinderlose Frauen und Männer womöglich stärker akzeptiert werden. Tab. 1 bestätigt zumindest, dass die Wohnsituation in der Stadt und dabei vor allem im Großraum Wien häufiger als Hindernis für den Kinderwunsch angesehen wird als in ländlichen Regionen. ${ }^{8}$

Im empirischen Teil geben wir einleitend den Kinderwunsch bei der Erstbefragung an (3406 Personen zwischen 21 und 45 Jahren). Das Hauptaugenmerk dieser Studie liegt jedoch auf der Realisierung des Drei-Jahres-Kinderwunsches. Dafür untersuchen wir jene 943 Männer und Frauen, die in beiden Erhebungswellen teilnahmen und in der ersten Welle angaben, sich innerhalb der nächsten drei Jahre ein Kind zu wünschen. Zum Zeitpunkt der Erstbefragung lebten 236 dieser Personen

\footnotetext{
8 Obwohl es bei diesem Argument nicht um die Zusammensetzung der Bevölkerung mit Kinderwunsch geht, sei erwähnt, dass im Analysesample der Personen mit Kinderwunsch die Befragten in Wien älter und häufiger kinderlos sind. Bei den Anteilen der unverheiratet zusammenlebenden Personen ergeben sich keine regionalen Unterschiede (Tab. 1).
} 
im Großraum Wien, 344 in anderen urbanen Regionen Österreichs und 363 in ländlichen Regionen. Aufgrund von fehlenden Angaben in den unabhängigen Variablen (z.B. Einstellungen zur Elternschaft) ist das Sample für die Regressionsmodelle etwas kleiner $(N=917)$.

Die Analyse des Kinderwunsches erfolgt in mehreren Schritten: Zuerst werden deskriptive Ergebnisse gezeigt. In einem zweiten Schritt werden binäre und multinomiale logistische Regressionen gerechnet. Dabei werden regionale Unterschiede bei der Verwirklichung des Kinderwunsches zunehmend detaillierter betrachtet. Die erste Modellvariante unterscheidet zwischen dem Großraum Wien und anderen Regionen und untersucht, ob der Kinderwunsch realisiert wurde oder nicht (binär). In der zweiten Variante wird zwischen Realisierung, Aufschub und Aufgabe des Kinderwunsches unterschieden (multinomial). In der dritten Variante wird zusätzlich zwischen urbanen und ländlichen Regionen differenziert. Ein Ausgangsmodell M1 beinhaltet jeweils nur die Region als erklärende Variable, ein erweitertes Modell M2 inkludiert die zuvor angegebenen Kovariaten. ${ }^{9}$ Um zu zeigen, ob die Hinzunahme von Kovariaten die Unterschiede zwischen dem Großraum Wien und den anderen Regionen ändert, wenden wir die von Karlson et al. (2012) entwickelte Methode an (KHB-Test). ${ }^{10}$ Falls dies zutrifft, kann angenommen werden, dass regionale Unterschiede in den zusätzlich aufgenommenen Variablen für regionale Unterschiede bei der Realisierung des Kinderwunsches verantwortlich sind. Von paritätsspezifischen Analysen wurde (mit Ausnahme der deskriptiven Analysen des Kinderwunsches) abgesehen, da nach Parität differenzierte Regressionsmodelle zur Analyse der Realisierung bestehender Kinderwünsche aufgrund der geringen Fallzahlen innerhalb der Regionen nicht geschätzt werden können. Der Fokus dieser Arbeit liegt jedoch auf regionalen und nicht auf paritätsspezifischen Unterschieden.

Abschließend erörtern wir die Effekte verschiedener sozio-demographischer und ökonomischer Charakteristika sowie von Einstellungen und Werten. Dafür wird zum einen das Modell für das gesamte Sample herangezogen und zwischen Realisierung, Aufschub und Aufgabe des Kinderwunsches unterschieden. Zum anderen werden separate Regressionsmodelle für den Großraum Wien, urbane Regionen und ländliche Regionen geschätzt, die aufgrund der geringeren Fallzahlen nur zwischen Realisierung und Nicht-Realisierung differenzieren. Anhand der von Hoetker (2007) vorgeschlagenen Methode testen wir, ob die berücksichtigten Charakteristika in allen Regionen dieselben Effekte auf die Verwirklichung des Kinderwunsches haben.

In den binären und multinomialen logistischen Regressionsmodellen werden sogenannte ,average marginal effects (AMEs)“ berechnet. Sie geben den durchschnittlichen Einfluss einer Variable auf die Wahrscheinlichkeit einer Realisierung (bzw. eines Aufschubs oder einer Aufgabe) des Kinderwunsches an. Der Vorteil von AMEs

\footnotetext{
${ }^{9}$ Ein weiteres Modell (M3) berücksichtigt auch den Erwerbsstatus und die Sicherheit des Kinderwunsches (hier nicht dargestellt). Obwohl sich Modell M3 einem LR-Test zufolge signifikant von Modell M2 unterscheidet, werden die hier dargestellten Ergebnisse davon nicht beeinflusst. Die Unterschiede in den Koeffizienten sind zwischen den Modellen auch nicht statistisch signifikant.

${ }^{10}$ Der KHB-Test basiert auf dem bekannten Sobel-Test zur Prüfung von Mediations-Effekten. In LogitModellen ist eine spezielle Methode notwendig, da die Varianz der zugrundeliegenden latenten Variablen in verschiedenen Modellen unterschiedlich ist. Deshalb kann eine Änderung in den geschätzten Koeffizienten nicht unmittelbar auf die Hinzunahme weiterer Variablen zurückgeführt werden (Karlson et al. 2012).
} 
ist die Vergleichbarkeit über verschiedene Modelle hinweg (vgl. Best und Wolf 2012). Positive Koeffizienten zeigen an, dass die entsprechende Gruppe öfter den Kinderwunsch realisierte (bzw. aufschob oder aufgab), negative Koeffizienten stehen für seltenere Realisierung (bzw. Aufschub oder Aufgabe).

\title{
4 Empirische Befunde
}

\subsection{Kinderwünsche}

Drei von zehn Männern und Frauen wünschten sich 2008/2009 innerhalb der nächsten drei Jahre ein Kind, zwei von zehn wollten später ein (weiteres) Kind und die Hälfte wollte keine (weiteren) Kinder mehr. Regionale Unterschiede erweisen sich als gering: Im Großraum Wien wünschten sich $30 \%$ innerhalb der nächsten drei Jahre ein Kind, in den urbanen Regionen $29 \%$ und in ländlichen Regionen $27 \%$ (Abb. 1). Zwischen 21 und $22 \%$ wollten später ein Kind bzw. weitere Kinder. Folglich hatten ähnlich viele die Familienplanung auch bereits abgeschlossen (48 bis $51 \%)$. Der Wunsch nach einem Kind in der nahen Zukunft war in den drei regionalen Räumen unter Kinderlosen und Eltern ähnlich häufig (rund 40 bis $42 \%$ bzw. 18 bis $20 \%)$.

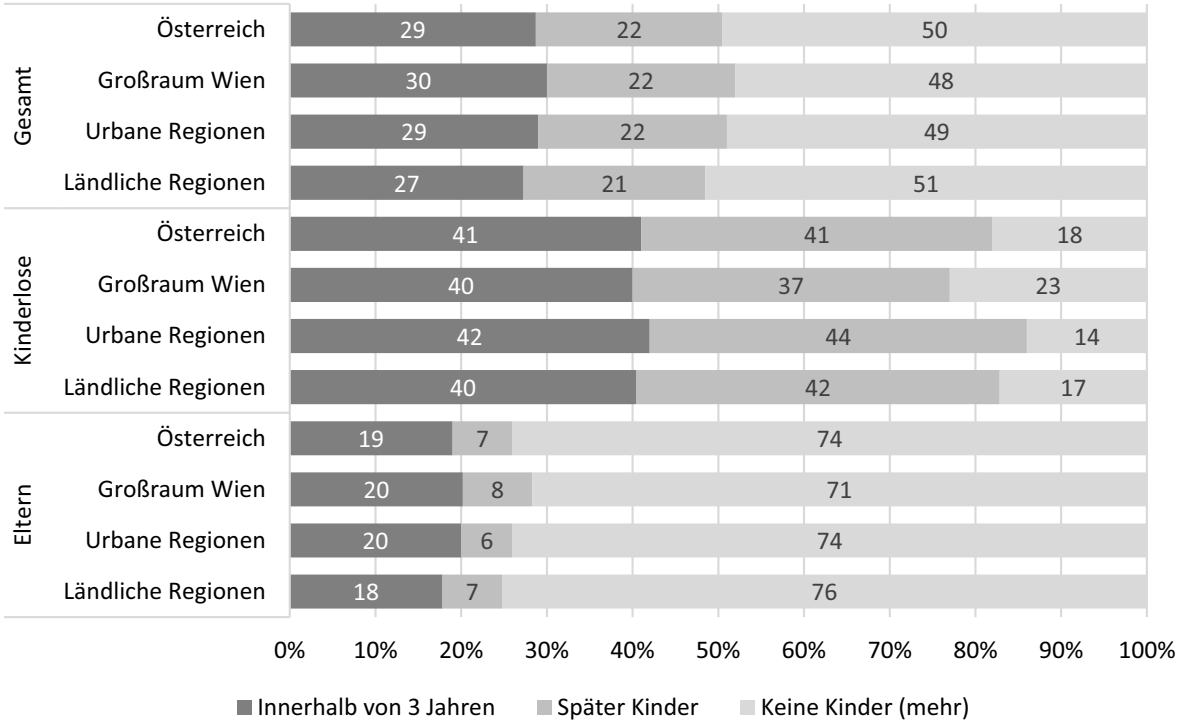

\begin{abstract}
Abb. 1 Kinderwunsch in der Erstbefragung. (Der „Großraum Wien“ umfasst Wien und das Wiener Umland (die Bezirke Korneuburg, Tulln, Bruck an der Leitha, Baden und Mödling sowie Teile der Bezirke Gänserndorf, Mistelbach und Wien-Umgebung (Statistik Austria 2015). Als urbane Regionen werden Orte mit hoher oder mittlerer Bevölkerungsdichte außerhalb des Großraums Wien bezeichnet (z. B. Linz, Graz, Salzburg, Innsbruck). Ländliche Regionen sind Orte mit niedriger Bevölkerungsdichte (weniger als 150 Einwohner pro $\mathrm{km}^{2}$ ). Quelle: GGS Welle 1; $N=3406$ Männer und Frauen im Alter von 21-45 Jahren. Gewichtete Werte)
\end{abstract}




\subsection{Verwirklichung des Drei-Jahres-Kinderwunsches}

Österreichweit verwirklichten vier von zehn ihren Drei-Jahres-Kinderwunsch (Abb. 2). Ebenfalls vier von zehn schoben ihren Kinderwunsch auf und wünschten sich weiter ein Kind, während zwei von zehn ihren Kinderwunsch aufgegeben hatten und sich kein (weiteres) Kind mehr wünschten. Die Realisierung kurzfristiger Kinderwünsche variiert regional: Im Großraum Wien verwirklichte ein Drittel den Drei-Jahres-Kinderwunsch, in den anderen Regionen waren es mit jeweils über $40 \%$ deutlich mehr. Die Familiengründung bzw. -erweiterung wurde im Großraum Wien dafür etwas häufiger verschoben (45 gegenüber 41 und $42 \%$ ) und mit $23 \%$ deutlich öfter aufgegeben als in anderen urbanen und in ländlichen Regionen, wo 18 bzw. $15 \%$ bei der Wiederbefragung kein (weiteres) Kind mehr wollten (Abb. 2).

Statistische Tests belegen unsere deskriptiven Befunde: Die signifikanten Ergebnisse in den Ausgangsmodellen in Tab. 2 sprechen dafür, dass der Kinderwunsch im Großraum Wien seltener realisiert wurde als in anderen Regionen Österreichs (Panel a, M1) und dass Männer und Frauen im Großraum Wien ihren Kinderwunsch öfter aufgegeben haben als jene in den anderen Regionen Österreichs (Panel b, M2). Zudem besteht vor allem ein Unterschied zwischen dem Großraum Wien und den anderen, urbanen wie ländlichen Regionen (Panel c, M1 und M2).

Werden sozioökonomische Charakteristika miteinbezogen, so unterscheiden sich Personen im Großraum Wien nicht mehr signifikant von jenen in urbanen oder ländlichen Regionen: KHB-Tests bestätigen, dass die geschätzten Unterschiede zwischen den Regionen unabhängig von der Modellspezifikation in Modell M2 kleiner sind als in Modell M1 (siehe Tab. 2, Panels a, b und c). Konkret zeigt sich in den KHBTests (Panels b und c), dass die in Modell M2 einbezogenen Charakteristika den im Großraum Wien häufigeren Aufschub erklären können, nicht aber Unterschiede bei der Aufgabe des Kinderwunsches. Somit ist die im Großraum Wien niedrigere Realisierungswahrscheinlichkeit zumindest zum Teil auf eine unterschiedliche $\mathrm{Zu}$ sammensetzung der Populationen, d.h. Unterschiede in sozio-demographischen und

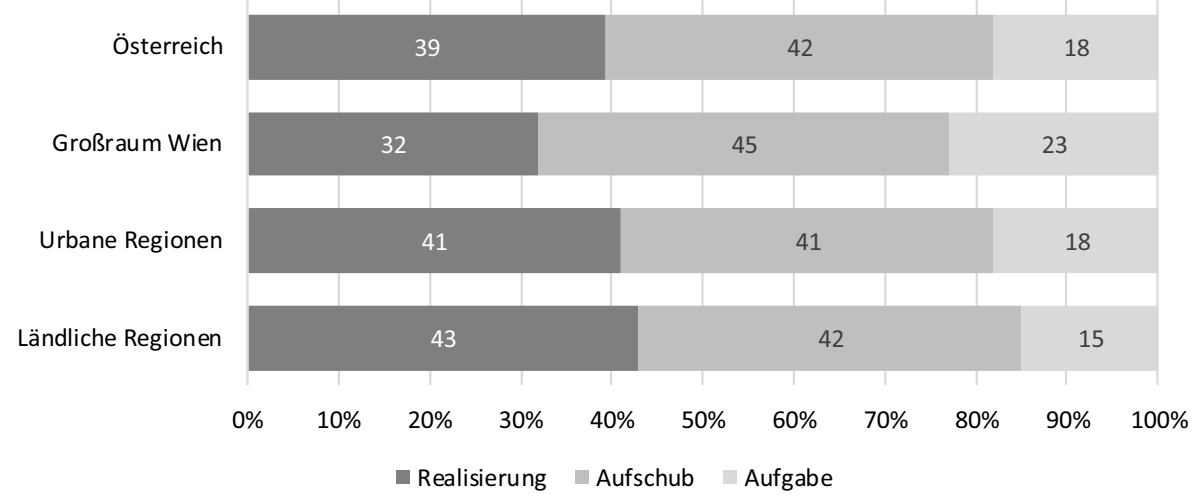

Abb. 2 Realisierung des Drei-Jahres-Kinderwunsches nach Region. (Für Definitionen des Großraums Wien, der urbanen und ländlichen Regionen siehe Abb. 1. Quelle: GGS Welle 1 und 2; N=943 Panelbefragte, die zum Zeitpunkt der ersten Erhebung binnen drei Jahre ein Kind haben wollten. Gewichtete Werte) 
Tab. 2 Regionale Unterschiede bei Verwirklichung, Aufschub und Aufgabe des Kinderwunsches (average marginal effects)

\begin{tabular}{lll}
\hline Modell & M1 & M2 \\
\hline
\end{tabular}

(a) Binomiale logistische Regression: Realisierung des Kinderwunsches

Realisierung (dichotom)

Großraum Wien

$-0,08^{\dagger}$

$-0,04^{\mathrm{a}}$

Andere Regionen (Referenz)

0

0

Cragg-Uhler $R^{2}$

0,01

0,23

(b) Multinomiale Regression: Realisierung, Aufschub und Aufgabe

Realisierung

Großraum Wien

$-0,08^{\dagger}$

$-0,04^{b}$

Andere Regionen (Referenz)

0

0

Aufschub

Großraum Wien

0,03

$-0,02^{\mathrm{b}}$

Andere Regionen (Referenz)

0

0

Aufgabe

Großraum Wien

0,05

$0,06^{*}$

Andere Regionen (Referenz)

0

0

Cragg-Uhler $R^{2}$

0,01

0,35

(c) Multinomiale Regression: Realisierung, Aufschub und Aufgabe

\section{Realisierung}

Großraum Wien

$-0,10 *$

$-0,05^{\mathrm{c}}$

Urbane Regionen

0,00

0,01

Ländliche Regionen (Referenz)

0

0

Aufschub

Großraum Wien

0,04

$-0,01^{\mathrm{c}}$

Urbane Regionen

0,00

0,01

Ländliche Regionen (Referenz)

0

0

Aufgabe

Großraum Wien

$0,06^{\dagger}$

$0,06^{\dagger}$

Urbane Regionen

0,00

0,00

Ländliche Regionen (Referenz)

Cragg-Uhler $R^{2}$

0

0

0,01

0,35

$N=917$. Für Definitionen des Großraums Wien, der urbanen und ländlichen Regionen siehe Abb. 1

Quelle: GGS Welle 1 und 2; Panelbefragte, die zum Zeitpunkt der ersten Erhebung binnen drei Jahre ein Kind haben wollten

$\dagger p \leq 0,10 ; * p \leq 0,05 ; * * p \leq 0,01 ; * * * p \leq 0,001$

Ergebnisse der KHB-Tests:

$\left.{ }^{\mathrm{a} U n t e r s c h i e d ~ b e i ~ R e a l i s i e r u n g / n i c h t-R e a l i s i e r u n g ~ z w i s c h e n ~ M o d e l l ~ M 1 ~ u n d ~ M o d e l l ~ M 2 ~(~} p \leq 0,05\right)$

${ }^{\mathrm{b}}$ Unterschied bei Realisierung/Aufschub zwischen Modell M1 und Modell M2 ( $\left.p \leq 0,01\right)$

${ }^{c}$ Unterschied bei Realisierung/Aufschub zwischen Großraum Wien und ländlichen Regionen zwischen Modell M1 und Modell M2 $(p \leq 0,05)$ 
ökonomischen Charakteristika sowie Wertvorstellungen zwischen den Menschen am Land und in der Stadt zurückzuführen.

\subsection{Regionale Gemeinsamkeiten und Unterschiede bei der Realisierung}

Erwartungsgemäß sind Alter, Partnerschaft und Parität wesentlich für die Realisierung von Drei-Jahres-Kinderwünschen (Tab. 3). Das gilt gleichermaßen für den Großraum Wien, urbane und ländliche Regionen. Im fortgeschrittenen reproduktiven Alter werden Kinderwünsche seltener realisiert. Personen in LAT-Partnerschaften realisierten zudem weniger oft ihre Kinderwünsche als Männer und Frauen, die zusammenlebten. Interessanterweise zeigen sich keine Unterschiede nach dem Familienstand: Unverheiratet Zusammenlebende realisierten ihren Kinderwunsch in gleichem Ausmaß wie Verheiratete. Wenig überraschend haben Personen, die in der Erstbefragung partnerlos waren und sich innerhalb der nächsten drei Jahre ein Kind wünschten, selten ihren Kinderwunsch realisiert. Auch die (bisherige) Anzahl der Kinder war von Bedeutung: Im Durchschnitt haben Personen mit einem Kind ihre Kinderwünsche öfter realisiert als etwa Kinderlose. Weiterführende Analysen weisen darauf hin, dass das auf eine höhere Realisierung von Frauen zurückzuführen sein könnte, die in der Erstbefragung ein Kind hatten und mit diesem Kind in Karenz waren. Mit der Realisierung ihres Kinderwunsches hatten sie somit zwei Kinder im Abstand von drei bis fünf Jahren. ${ }^{11}$ Eltern mit zwei oder mehr Kindern hatten ihre gewünschte Familienerweiterung öfters bereits aufgegeben.

Auch die Bildung erweist sich als relevant. Personen mit tertiärer Bildung gaben Kinderwünsche seltener auf als Personen mit Primär- oder Sekundarbildung (Tab. 3). Darüber hinaus kommt es zu signifikanten regionalen Unterschieden: In Wien realisierten höher Gebildete ihren Kinderwunsch öfter als Personen ohne tertiären Bildungsabschluss (Tab. 4). Auch in urbanen Regionen ist dieser Zusammenhang zu erkennen, er ist jedoch statistisch nicht signifikant. Im ländlichen Raum ist höhere Bildung hingegen nicht mit einer größeren Realisierungschance verbunden.

Bezüglich finanzieller Einschränkungen zeigt sich, dass in Österreich eine gute ökonomische Situation mit einer häufigeren Realisierung des Kinderwunsches assoziiert ist. Bei finanziellen Problemen wird der Kinderwunsch vermehrt aufgeschoben. Dies scheint vor allem auf dem Land zuzutreffen (Tab. 4). Der Unterschied zwischen den Regionen ist jedoch statistisch nicht signifikant.

Familienbezogene Werte und Einstellungen sind ebenfalls relevant. Personen, die Kinder als notwendig für ein erfülltes Leben ansehen, realisierten ihren Kinderwunsch häufiger und gaben ihn im Gegenzug dafür seltener auf. In den separaten Berechnungen für die Regionen ist dieser Effekt nur in den urbanen Regionen deutlich (Graz, Linz, Salzburg etc.) zu erkennen. Dennoch gibt es keine statistisch signifikanten Unterschiede zwischen dem Großraum Wien, den urbanen und den

\footnotetext{
11 Personen in Karenz realisierten ihren Kinderwunsch generell häufiger und schoben ihn im Gegenzug seltener auf. Karenzierte Personen mit einem Kind verwirklichten ihren Kinderwunsch häufiger als kinderlose Personen, die in Beschäftigung waren. Personen in Karenz, die bereits zwei oder mehr Kinder hatten, verhielten sich ähnlich wie erwerbstätige Personen mit einem Kind: Sie schoben ihren Kinderwunsch im Vergleich zu den berufstätigen Kinderlosen nicht nur seltener auf, sondern gaben ihn auch häufiger auf.
} 
Tab. 3 Einflussfaktoren der Verwirklichung, des Aufschubs und der Aufgabe des Kinderwunsches (average marginal effects)

\begin{tabular}{|c|c|c|c|}
\hline \multirow{2}{*}{$\begin{array}{l}\text { Multinomiale logistische Regression } \\
\text { Modell M2 }\end{array}$} & \multicolumn{3}{|l|}{ Österreich } \\
\hline & Realisierung & Aufschub & Aufgabe \\
\hline \multicolumn{4}{|l|}{ Region } \\
\hline Großraum Wien & $-0,05$ & $-0,01$ & $0,06^{\dagger}$ \\
\hline Urbane Regionen & 0,01 & $-0,01$ & 0,00 \\
\hline Ländliche Regionen (Referenz) & 0 & 0 & 0 \\
\hline \multicolumn{4}{|l|}{ Geschlecht } \\
\hline Männlich (Referenz) & 0 & 0 & 0 \\
\hline Weiblich & 0,03 & $-0,06^{*}$ & 0,03 \\
\hline \multicolumn{4}{|l|}{ Alter } \\
\hline 21-24 Jahre & 0,03 & 0,07 & $-0,10 * * *$ \\
\hline 25-34 Jahre (Referenz) & 0 & 0 & 0 \\
\hline 35-45 Jahre & $-0,27 * * *$ & 0,03 & $0,24 * * *$ \\
\hline \multicolumn{4}{|l|}{ Partnerschaftsstatus } \\
\hline Verheiratet (Referenz) & 0 & 0 & 0 \\
\hline Unverheiratet zusammenlebend & $-0,04$ & 0,06 & $-0,02$ \\
\hline LAT & $-0,22 * * *$ & $0,22 * * *$ & $-0,01$ \\
\hline Kein/e Partner/in & $-0,31 * * *$ & $0,23 * * *$ & $0,07^{\dagger}$ \\
\hline \multicolumn{4}{|l|}{ Elternschaft (Parität) } \\
\hline Kinderlos (Referenz) & 0 & 0 & 0 \\
\hline $1 \mathrm{Kind}$ & $0,08^{*}$ & $-0,15^{* * *}$ & $0,07 *$ \\
\hline 2 Kinder & 0,02 & $-0,26 * * *$ & $0,24 * * *$ \\
\hline 3 oder mehr Kinder & $-0,04$ & $-0,12$ & $0,16^{*}$ \\
\hline \multicolumn{4}{|l|}{ Höchster Bildungsabschluss } \\
\hline Primär- oder Sekundärbildung (Referenz) & 0 & 0 & 0 \\
\hline Tertiärer Bildungsabschluss & 0,06 & 0,02 & $-0,08 * *$ \\
\hline Unbekannt & $-0,01$ & 0,12 & $-0,12 * *$ \\
\hline \multicolumn{4}{|l|}{ Ökonomische Lage } \\
\hline Situation (sehr) schwierig (Referenz) & 0 & 0 & 0 \\
\hline Situation ok & $0,08^{*}$ & $-0,08^{*}$ & 0,00 \\
\hline Situation (sehr) gut & $0,13 * *$ & $-0,12 * *$ & $-0,01$ \\
\hline \multicolumn{4}{|l|}{ Einstellung zur Elternschaft } \\
\hline Kind(er) notwendig für ein erfülltes Leben & $0,10^{*}$ & $-0,03$ & $-0,06^{\dagger}$ \\
\hline Weder/noch (Referenz) & 0 & 0 & 0 \\
\hline Kind(er) nicht notwendig & 0,00 & 0,01 & $-0,01$ \\
\hline \multicolumn{4}{|l|}{ Geburtsland } \\
\hline Österreich (Referenz) & 0 & 0 & 0 \\
\hline Anderes Land & $-0,04$ & 0,06 & $-0,02$ \\
\hline \multicolumn{4}{|c|}{ Wohnverhältnisse beeinflussen Entscheidung für Kind } \\
\hline ... keineswegs (Referenz) & 0 & 0 & 0 \\
\hline ... ein bisschen & $-0,05$ & $0,07^{\dagger}$ & $-0,02$ \\
\hline ... stark & $-0,07^{\dagger}$ & 0,04 & 0,03 \\
\hline
\end{tabular}


Tab. 3 (Fortsetzung)

\begin{tabular}{llll}
\hline $\begin{array}{l}\text { Multinomiale logistische Regression } \\
\text { Modell M2 }\end{array}$ & $\begin{array}{l}\text { Österreich } \\
\text { Realisierung }\end{array}$ & Aufschub & Aufgabe \\
\hline $\begin{array}{l}\text { Häufigkeit des Kirchgangs (o. A.) } \\
\text { Nie (Referenz) }\end{array}$ & 0 & 0 & 0 \\
Weniger als einmal im Monat & $-0,04$ & $0,07^{\dagger}$ & $-0,03$ \\
Einmal im Monat oder öfter & 0,00 & 0,03 & $-0,03$ \\
\hline
\end{tabular}

$N=917$. Das hier dargestellte Modell entspricht Modell M2 in Panel (c) in Tab. 2. Für Definitionen des Großraums Wien, der urbanen und ländlichen Regionen siehe Abb. 1

Quelle: GGS Welle 1 und 2; Panelbefragte, die zum Zeitpunkt der ersten Erhebung binnen drei Jahre ein Kind haben wollten

${ }^{\dagger} p \leq 0,10 ; * p \leq 0,05 ; * * p \leq 0,01 ; * * * p \leq 0,001$

Tab. 4 Realisierung des Kinderwunsches nach Region (average marginal effects)

\begin{tabular}{|c|c|c|c|}
\hline Binäre logistische Regression & Realisierung & & \\
\hline Modell M2 & $\begin{array}{l}\text { Großraum } \\
\text { Wien }\end{array}$ & $\begin{array}{l}\text { Urbane Regio- } \\
\text { nen }\end{array}$ & $\begin{array}{l}\text { Ländliche } \\
\text { Regionen }\end{array}$ \\
\hline \multicolumn{4}{|l|}{ Höchster Bildungsabschluss } \\
\hline $\begin{array}{l}\text { Primär- oder Sekundärbildung (Refe- } \\
\text { renz) }\end{array}$ & 0 & 0 & 0 \\
\hline Tertiärer Bildungsabschluss & $0,15^{*}$ & 0,08 & $-0,04^{\mathrm{b}}$ \\
\hline Unbekannt & 0,07 & $-0,03$ & 0,09 \\
\hline \multicolumn{4}{|l|}{ Ökonomische Lage } \\
\hline Situation (sehr) schwierig (Referenz) & 0 & 0 & 0 \\
\hline Situation ok & $-0,04$ & 0,04 & $0,20 * *$ \\
\hline Situation (sehr) gut & 0,00 & 0,11 & $0,21 * *$ \\
\hline \multicolumn{4}{|l|}{ Einstellung zur Elternschaft } \\
\hline $\begin{array}{l}\text { Kind(er) notwendig für ein erfülltes } \\
\text { Leben }\end{array}$ & 0,03 & $0,17^{*}$ & 0,07 \\
\hline Weder/noch (Referenz) & 0 & 0 & 0 \\
\hline Kind(er) nicht notwendig & $-0,02$ & 0,00 & $-0,03$ \\
\hline \multicolumn{4}{|c|}{ Wohnverhältnisse beeinflussen Entscheidung für Kind } \\
\hline ... keineswegs & 0 & 0 & 0 \\
\hline ... ein bisschen & $-0,05$ & $-0,08$ & $-0,04$ \\
\hline ... stark & $-0,16^{*}$ & $-0,06^{\mathrm{c}}$ & $-0,01^{\mathrm{c}}$ \\
\hline Cragg-Uhler $R^{2}$ & 0,40 & 0,27 & 0,22 \\
\hline$N$ & 227 & 333 & 357 \\
\hline
\end{tabular}

Kontrolliert über Geschlecht, Alter, Partnerschaftsstatus, Migrationshintergrund und Religiosität. Für Definitionen des Großraums Wien, der urbanen und ländlichen Regionen siehe Abb. 1

Quelle: GGS Welle 1 und 2; Panelbefragte, die zum Zeitpunkt der ersten Erhebung binnen drei Jahre ein Kind haben wollten.

${ }^{\dagger} p \leq 0,10 ; * p \leq 0,05 ; * * p \leq 0,01 ; * * * p \leq 0,001$.

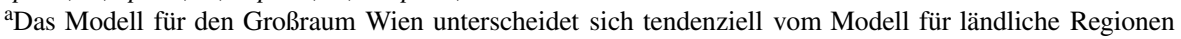
$\left(\right.$ LR Chi $\left.{ }^{2}=28,61 ; \mathrm{df}=19 ; p \leq 0,10\right)$

${ }^{b}$ Der Unterschied nach dem höchsten Bildungsabschluss unterscheidet sich tendenziell zwischen dem Großraum Wien und den ländlichen Regionen (LR Chi ${ }^{2}=2,77 ; \mathrm{df}=1 ; p \leq 0,10$ )

${ }^{c}$ Der Unterschied nach Wohnverhältnissen (starker Einfluss) variiert sowohl zwischen dem Großraum Wien und anderen urbanen Regionen ( $\mathrm{LR} \mathrm{Chi}^{2}=4,71$; $\left.\mathrm{df}=1 ; p \leq 0,05\right)$ als auch zwischen dem Großraum Wien und den ländlichen Regionen $\left(\mathrm{LR} \mathrm{Chi}^{2}=2,87 ; \mathrm{df}=1 ; p \leq 0,10\right)$ 
ländlichen Regionen. Zuwanderung oder Religiosität scheinen nur von geringer Bedeutung für Realisierung, Aufschub oder Aufgabe des Kinderwunsches zu sein.

Die separaten Analysen für den Großraum Wien, urbane und ländliche Regionen ergaben zudem, dass die Wohnverhältnisse nur im Großraum Wien mit der Realisierung des Kinderwunsches assoziiert sind. Werden die Wohnverhältnisse stark mit der Entscheidung für oder gegen ein (weiteres) Kind verknüpft, so wirkt sich dies bei den WienerInnen negativ auf die Realisierungswahrscheinlichkeit aus. Auch in urbanen Regionen ist dieser negative Zusammenhang zu erkennen, er ist jedoch statistisch nicht signifikant. Im ländlichen Raum scheinen die Wohnverhältnisse hingegen ein weniger bedeutendes Hindernis für die Verwirklichung des Kinderwunsches darzustellen.

\section{Diskussion}

Der vorliegende Aufsatz belegt die Bedeutung des urbanen Raums für die Verwirklichung des Kinderwunsches. Obwohl sich der Drei-Jahres-Kinderwunsch selbst kaum unterschied, war dessen Realisierung im Großraum Wien deutlich geringer als in anderen urbanen oder den ländlichen Regionen. Analysen regionaler Unterschiede im Fertilitätsverhalten beschränkten sich bislang vorrangig auf den Übergang zur Elternschaft, die Geburt eines zweiten Kindes sowie die Zahl geborener Kinder (Bocquier und Costa 2015; Kulu und Washbrook 2014). Unsere Studie zeigt, dass auch bei der Umsetzung des Kinderwunsches das regionale Umfeld relevant ist.

Unterschiede in der Bevölkerungszusammensetzung (Alter, Bildung, Partnerschaftsstatus etc.) konnten erklären, warum Personen in Wien den Kinderwunsch häufiger aufschieben. Dies bestätigt Arbeiten, die die unterschiedliche Bevölkerungsstruktur als zentral für regionale Fertilitätsunterschiede ansehen (Hank 2002; Trovato und Grindstaff 1980). Für das häufigere Aufgeben des Drei-Jahres-Kinderwunsches bestehen aber auch nach Einbeziehung demographischer Charakteristika sowie Wertvorstellungen noch regionale Unterschiede. Dafür könnte die Vielfalt an bildungs- und berufsbezogenen Optionen in Städten verantwortlich sein, die mit einer Familiengründung oder Familienerweiterung konkurrieren.

Nicht zuletzt zeigte sich, dass der formale Bildungsgrad in der Stadt, nicht aber am Land relevant für die Umsetzung des Kinderwunsches ist. Für die höhere Realisierung des Kinderwunsches von tertiär Gebildeten im Großraum Wien könnten die besseren Betreuungsmöglichkeiten für Kinder - insbesondere für die Jüngsten - relevant sein (Blum et al. 2014; Statistik Austria 2019b). Sie sind zentral für die Vereinbarkeit von Familie und Beruf und damit speziell für die Opportunitätskosten höher gebildeter Frauen.

Auch die Wohnverhältnisse erwiesen sich vor allem als wesentlich für die Realisierung im Großraum Wien. Unsere Ergebnisse befinden sich damit nicht nur in Einklang mit der Literatur zur Relevanz des Wohnumfelds für Familiengründungen (z. B. Vignoli et al. 2013), sondern weisen darüber hinaus auch auf die besondere Bedeutung des Wohnumfelds im städtischen Raum hin. Zukünftige Studien sollten die unterschiedliche Wohnsituation im städtischen und ländlichen Raum in Österreich (Statistik Austria 2018) noch detaillierter berücksichtigen. 
Eine Longitudinalstudie zur Fertilität wurde in Österreich bisher erst einmal durchgeführt (1978 und 1983; vgl. Gisser et al. 1985). Die Gruppe der jungen verheirateten Frauen, die sich in der Erstbefragung ein Kind gewünscht und die bis zur Wiederbefragung eines bekommen hatten, umfasste damals rund $40 \%$. Eine Einschränkung der GGS-Paneldaten der Jahre 2008/2009 und 2012/2013 auf eine ähnliche Vergleichsgruppe (i.e. Frauen, die weniger als fünf Jahre mit ihrem Partner zusammenlebten und zum Zeitpunkt des Zusammenziehens oder der Heirat unter 30 waren) zeigt, dass die Gruppe „Kind gewünscht und bekommen“ damals wie heute annähernd gleich groß war (Buber-Ennser und Gisser 2013). In der Studie in den 1980er-Jahren war ein deutliches Stadt-Land-Gefälle ersichtlich. Frauen in ländlichen Gebieten erfüllten ihre Kinderwünsche eher als Frauen im Kernraum Wien. Dafür mitverantwortlich war, dass der Beruf des Mannes und die Berufsorientierung der Frau sich zwischen Wien und den ländlichen Regionen deutlich unterschieden: Frauen von Landwirten hatten ihre Kinderwünsche ,übererfüllt“ (hatten bei der Wiederbefragung mehr Kinder als in der Erstbefragung gewollt), berufsorientierte Frauen hatten sie ,untererfüllt" (hatten bei der Wiederbefragung weniger Kinder als in der Erstbefragung gewollt) (siehe Gisser et al. 1985, S. 86). Unsere Studie zeigt nun, dass regionale Unterschiede bei der Realisierung des Kinderwunsches drei Jahrzehnte später nach wie vor bestehen, obwohl der landwirtschaftliche Sektor inzwischen stark an Bedeutung verloren hat (Statistik Austria 2019a).

Das Kinderkriegen verläuft freilich nicht immer intentional. Zudem ändern Individuen und Paare ihre Kinderwünsche im Zeitverlauf (Hayford 2009; Iacovou und Tavares 2011). Unter den Personen, die 2008/2009 angaben, erst später Kinder zu wollen, hatten $9 \%$ bis zur Zweitbefragung - und somit früher als in der Erstbefragung angegeben - ein Kind bekommen. Dies war in ländlichen und urbanen Regionen häufiger als im Großraum Wien (10, 9 bzw. 7\%). Auch war nur ein geringer Anteil derer, die ursprünglich keine weiteren Kinder mehr planten, bis zur Wiederbefragung (erneut) Eltern geworden: $4 \%$ österreichweit, $2 \%$ im Großraum Wien, $5 \%$ in urbanen und $4 \%$ in ländlichen Regionen. Diese beiden Gruppen standen jedoch nicht im Fokus der vorliegenden Studie (wir verweisen dazu weiter auf Brzozowska et al. 2018).

Der persönliche Kinderwunsch ist zwar ein individuelles Merkmal, doch sind am Zustandekommen eines Kindes zwei Personen beteiligt, weshalb zumindest bei bestehenden Partnerschaften die Charakteristika beider von Bedeutung sind (Corijn et al. 1996). Obwohl der GGS Informationen zum/zur im Haushalt lebenden Partner bzw. Partnerin beinhaltet, ist die vorliegende Auswertung nicht paarbezogen. Da ein beträchtlicher Anteil der Personen mit konkreten Kinderwunsch-Plänen in LATPartnerschaften lebte oder partnerlos war (Tab. 1) und daher keine entsprechenden Informationen vorhanden sind, wurde auf eine paarbezogene Auswertung verzichtet.

Leider war es uns nicht möglich, alle wesentlichen Aspekte für die Realisierung von Kinderwünschen in die Untersuchung aufzunehmen, da entweder dafür geeignete Indikatoren in den Daten fehlten oder die Fallzahl eine nötige weitere Unterteilung des Samples in Subgruppen nicht erlaubte. Aus diesen Gründen konnte etwa nicht auf die Rolle der Kinderbetreuung (Aassve et al. 2012), die Konfessionszugehörigkeit, auf Umzüge (z.B. von dicht bebauten Gebieten oder urbanen Zentren in Vorstädte vor der Familiengründung) (Birg und Flöthmann 1990) oder den 
Unterschied zwischen der Stadt und ihren Vorstädten (Kulu und Boyle 2009) eingegangen werden. Darüber hinaus wäre es wünschenswert, unsere Befunde mit Daten aus anderen Ländern zu replizieren und Kontexteffekte des städtischen Umfeldes in Mehrebenenmodellen zu prüfen. Da heute mehr Menschen als je zuvor in nach wie vor wachsenden Städten leben, sollte der regionale Kontext in der Forschung zum Kinderwunsch und dessen Verwirklichung größere Beachtung finden.

Funding Open access funding provided by University of Vienna.

Open Access Dieser Artikel wird unter der Creative Commons Namensnennung 4.0 International Lizenz veröffentlicht, welche die Nutzung, Vervielfältigung, Bearbeitung, Verbreitung und Wiedergabe in jeglichem Medium und Format erlaubt, sofern Sie den/die ursprünglichen Autor(en) und die Quelle ordnungsgemäß nennen, einen Link zur Creative Commons Lizenz beifügen und angeben, ob Änderungen vorgenommen wurden.

Die in diesem Artikel enthaltenen Bilder und sonstiges Drittmaterial unterliegen ebenfalls der genannten Creative Commons Lizenz, sofern sich aus der Abbildungslegende nichts anderes ergibt. Sofern das betreffende Material nicht unter der genannten Creative Commons Lizenz steht und die betreffende Handlung nicht nach gesetzlichen Vorschriften erlaubt ist, ist für die oben aufgeführten Weiterverwendungen des Materials die Einwilligung des jeweiligen Rechteinhabers einzuholen.

Weitere Details zur Lizenz entnehmen Sie bitte der Lizenzinformation auf http://creativecommons.org/ licenses/by/4.0/deed.de.

\section{Literatur}

Aassve, Arnstein, Elena Meroni, und Chiara Pronzato. 2012. Grandparenting and childbearing in the extended family. European Journal of Population 28:499-518. https://doi.org/10.1007/s10680-012-92732.

Ajzen, Icek, und Jane Klobas. 2013. Fertility intentions: an approach based on the theory of planned behavior. Demographic Research 29(8):203-232. https://doi.org/10.4054/DemRes.2013.29.8.

Beham-Rabanser, Martina, Caroline Berghammer, und Ulrike Zartler. 2019. Partnerbeziehungen zwischen Flexibilität und Stabilität. In Sozialstruktur und Wertewandel in Österreich. Trends 1986-2016, Hrsg. Johann Bacher, Alfred Grausgruber, Max Haller, Franz Höllinger, Dimitri Prandner, und Roland Verwiebe, 179-209. Wiesbaden: Springer.

Berghammer, Caroline. 2012. Church attendance and childbearing: evidence from a Dutch panel study, 1987-2005. Population Studies 66(2):197-212. https://doi.org/10.1080/00324728.2012.655304.

Berghammer, Caroline, und Eva-Maria Schmidt. 2019. Familie, Partnerschaft und Geschlechterrollen: Alles im Wandel? In Quo vadis, Österreich? Wertewandel zwischen 1990 und 2018, Hrsg. Julia Aichholzer, Christian Friesl, Sanja Hajdinjak, und Sylvia Kritzinger, 57-88. Wien: Czernin.

Berghammer, Caroline, Martina Beham-Rabanser, und Ulrike Zartler. 2019. Machen Kinder glücklich? Wert von Kindern und ideale Kinderzahl. In Sozialstruktur und Wertewandel in Österreich. Trends 1986-2016, Hrsg. Johann Bacher, Alfred Grausgruber, Max Haller, Franz Höllinger, Dimitri Prandner, und Roland Verwiebe, 211-242. Wiesbaden: Springer.

Best, Henning, und Christof Wolf. 2012. Modellvergleich und Ergebnisinterpretation in Logit- und ProbitRegressionen. Kölner Zeitschrift für Soziologie und Sozialpsychologie 64:377-395. https://doi.org/ 10.1007/s11577-012-0167-4.

Birg, Herwig, und Ernst-Jürgen Flöthmann. 1990. Regionsspezifische Wechselwirkungen zwischen Fertilität und Migration im Lebenslauf. Acta Demographica 1:1-26.

Birg, Herwig, Ernst-Jürgen Flöthmann, und Iris Reiter. 1991. Biographische Theorie der demographischen Reproduktion. Frankfurt, New York: Campus.

Blum, Sonja, Lenka Formánková, und Ivana Dobrotić. 2014. Family policies in 'hybrid'welfare states after the crisis: Pathways between policy expansion and retrenchment. Social Policy \& Administration 48(4):468-491. https://doi.org/10.1111/spol.12071. 
BMSGK. 2004. Ein kindgerechtes Österreich. Nationaler Aktionsplan für die Rechte von Kindern und Jugendlichen. Wien: BMSGK.

Bocquier, Philippe, und Rafael Costa. 2015. Which transition comes first? Urban and demographic transitions in Belgium and Sweden. Demographic Research 33(48):1297-1332. https://doi.org/10.4054/ DemRes.2015.33.48.

Brzozowska, Zuzanna, Isabella Buber-Ennser, und Bernhard Riederer. 2018. Didn't plan one but got one: Mistimed and unintended births among men and women in six European countries. VID Working Paper 5/2018. https://www.oeaw.ac.at/fileadmin/subsites/Institute/VID/PDF/Publications/Working Papers/WP2018_05.pdf. Zugegriffen: 2. Nov. 2020.

Buber, Isabella. 2010. Parity-specific weights for the Austrian generations and gender survey. VID Working Paper 4/2010. Vienna: Institute of Demography.

Buber-Ennser, Isabella. 2014. Attrition in the Austrian Generations and Gender Survey: Is there a bias by fertility-relevant aspects? Demographic Research 31(16):459-496. https://doi.org/10.4054/DemRes. 2014.31.16

Buber-Ennser, Isabella, und Katrin Fliegenschnee. 2013. Being ready for a child. A mixed-methods investigation of fertility intentions. Family Science 4(1):139-147. https://doi.org/10.1080/19424620.2013. 871739 .

Buber-Ennser, Isabella, und Richard Gisser. 2013. Vergleich mit der ersten österreichischen Longitudinalstudie um 1980. In Familienentwicklung in Österreich 2009-2013. Partnerschaft, Kinderwunsch, Kinderbetreuung und ökonomische Situation, Bd. 35, Hrsg. Isabella Buber-Ennser, Norbert Neuwirth, und Maria Rita Testa. Vienna: VID/ÖIF.

Corijn, Martine, Aart C. Liefbroer, und Jenny de Jong Gierveld. 1996. It takes two to tango, doens't it? The influence of couple characteristics on the timing of the birth of the first child. Journal of Marriage and the Family 58:117-126.

Ferdinand, Ursula. 1999. Das Malthusische Erbe. Entwicklungsstränge der Bevölkerungstheorie im 19. Jahrhundert und deren Einfluß auf die radikale Frauenbewegung in Deutschland. Münster: LIT.

Ferrarini, Tommy. 2006. Families, states and labour markets: Institutions, causes and consequences of family policy in post-war welfare states. Cheltenham: Edward Elgar Publishing.

Galloway, Patrick R., D. Lee Ronald, und Eugene A. Hammel. 1998. Urban versus rural: fertility decline in the cities and rural districts of prussia, 1875 to 1910. European Journal of Population 14(3):209-264. https://doi.org/10.1023/a:1006032332021.

Gisser, Richard, Wolfgang Lutz, und Rainer Münz. 1985. Kinderwunsch und Kinderzahl. In Leben mit Kindern. Wunsch und Wirklichkeit, Hrsg. Rainer Münz, 33-93. Wien: Deuticke.

Goujon, Anne, Sandra Jurasszovich, und Michaela Potančoková. 2017. Religious denominations in Austria: baseline study for 2016-Scenarios until 2046. ÖIF-Forschungsbericht. Wien: Österreichischer Integrationsfonds.

Goujon, Anne, Vegard Skirbekk, Katrin Fliegenschnee, und Pawel Strzelecki. 2007. New times, old beliefs: Projecting the future size of religions in Austria. Vienna Yearbook of Population Research. https://doi. org/10.1553/populationyearbook2007s237.

Hank, Karsten. 2002. Regional social contexts and individual fertility decisions: a multilevel analysis of first and second births in western Germany. European Journal of Population 18(3):281-299. https:// doi.org/10.1023/A:1019765026537.

Hayford, S.R. 2009. The evolution of fertility expectations over the life course. Demography 46(4):765-783.

Hoetker, Glenn. 2007. The use of logit and probit models in strategic management research: Critical issues. Strategic Management Journal 28(4):331-343.

Iacovou, Maria, und Lara Patricio Tavares. 2011. Yearning, learning and conceding: reasons men and women change their childbearing intentions. Population and Development Review 37(1):89-123. https:// doi.org/10.1111/j.1728-4457.2011.00391.x.

Jaffe, Alfred J. 1942. Urbanization and fertility. American Journal of Sociology 48(1):48-60.

van de Kaa, Dirk J. 1987. Europe's second demographic transition. Population Bulletin 42(1):1-59.

Kapitány, Balázs, und Zsolt Spéder. 2012. Realisation, postponement or abandonment of fertility intentions in four European countries. Population-E 67(4):599-630. https://doi.org/10.3917/popu.1204.0711.

Karlson, Kristian Bernt, Anders Holm, und Richard Breen. 2012. Comparing regression coefficients between same-sample nested models using logit and probit: a new method. Sociological Methodology 42(1):286-313. https://doi.org/10.1177/0081175012444861.

Kaufmann, Franz-Xaver. 1990. Zukunft der Familie. Stabilität, Stabilitätsrisiken und Wandel der familialen Lebensformen sowie ihre gesellschaftlichen und politischen Bedingungen. München: Beck. 
Korpi, Walter. 2000. Faces of inequality: Gender, class, and patterns of inequalities in different types of welfare states. Social Politics: International Studies in Gender, State \& Society 7(2):127-191. https:// doi.org/10.1093/sp/7.2.127.

Kuhnt, Anne-Kristin, und Heike Trappe. 2016. Channels of social influence on the realization of shortterm fertility intentions in Germany. Advances in Life Course Research 27:16-29. https://doi.org/10. 1016/j.alcr.2015.10.002.

Kulu, Hill, und Paul J. Boyle. 2009. High fertility in city suburbs: compositional or contextual effects? European Journal of Population 25(2):157-174. https://doi.org/10.1007/s10680-008-9163-9.

Kulu, Hill, und Elizabeth Washbrook. 2014. Residential context, migration and fertility in a modern urban society. Advances in Life Course Research 21:168-182. https://doi.org/10.1016/j.alcr.2014.01.001.

Lesthaeghe, Ron. 1995. The second demographic transition in western countries: an interpretation. In Gender and family change in industrialized countries, Hrsg. Karen O. Mason, An-Magritt Jensen, 17-62. Oxford: Clarendon Press.

Mencarini, Letizia, Daniele Vignoli, und Anna Gottard. 2015. Fertility intentions and outcomes: implementing the theory of planned behavior with graphical models. Advances in Life Course Research 23:14-28. https://doi.org/10.1016/j.alcr.2014.12.004.

Mulder, Clara H. 2006. Home-ownership and family formation. Journal of Housing and the Built Environment 21(3):281-298.

Nauck, Bernhard. 2001. Der Wert von Kindern für ihre Eltern: „Value of Children“ als spezielle Handlungstheorie des generativen Verhaltens und von Generationenbeziehungen im interkulturellen Vergleich. Kölner Zeitschrift für Soziologie und Sozialpsychologie 53(3):407-435.

Neuwirth, Norbert, Andreas Baierl, Markus Kaindl, Christiane Rille-Pfeiffer, und Georg Wernhart. 2011. Der Kinderwunsch in Österreich. Umfang, Struktur und wesentliche Determinanten. Eine Analyse anhand des GGP. Forschungsbericht 5/2011. Wien: ÖIF.

OECD. 2009. Doing better for children. Paris: OECD Publishing.

OECD. 2011. OECD regional typology. Paris: Directorate for Public Governance and Territorial Development.

Philipov, Dimiter, und Caroline Berghammer. 2007. Religion and fertility ideals, intentions and behaviour: a comparative study of European countries. Vienna Yearbook of Population Research 2007:271-305. https://doi.org/10.1553/populationyearbook2007s271.

Prskawetz, Alexia, Tomás Sobotka, Isabella Buber, Henriette Engelhardt, und Richard Gisser. 2008. Austria: persistent low fertility since the mid-1980s. Demographic Research 19(12):293-360. https://doi. org/10.4054/DemRes.2008.19.12.

Régnier-Loilier, Arnaud, und Daniele Vignoli. 2011. Fertility intentions and obstacles to their realization in France and Italy. Population-E 66(2):361-390. https://doi.org/10.3917/popu.1102.0401.

Riederer, Bernhard. 2005. Tradition, Investitionsgut oder Herzenssache? Der Wunsch nach Kindern zwischen Ideal und Realität. In Österreich zur Jahrhundertwende. Gesellschaftliche Werthaltungen und Lebensqualität 1986-2004, Hrsg. Wolfgang Schulz, Max Haller, und Alfred Grausgruber, 367-400. Wiesbaden: VS.

Riederer, Bernhard. 2018. Elternschaft und Wohlbefinden. Kinder im individuellen, partnerschaftlichen und gesellschaftlichen Kontext. Wiesbaden: Springer.

Riederer, Bernhard, und Isabella Buber-Ennser. 2019. Regional context and realization of fertility intentions: the role of the urban context. Regional Studies 53(12):1669-1679. https://doi.org/10.1080/ 00343404.2019 .1599843 .

Riederer, Bernhard, Isabella Buber-Ennser, und Raimund Haindorfer. 2020. Trends in demography and migration in Austria. In Demography and migration in Central and Eastern Europe, Hrsg. Péter Tálas, Alex Etl, 9-27. Budapest: Dialóg Campus.

Riederer, Bernhard, Roland Verwiebe, und Lena Seewann. 2019. Changing social stratification in Vienna: why are migrants declining from the middle of society? Population, Space and Place 25(2):e2215. https://doi.org/10.1002/psp.2215.

Rinesi, Francesca, Antonella Pinnelli, Sabrina Prati, Cinzia Castagnaro, und Claudia Iaccarino. 2011. Avoir un deuxième enfant en Italie: de l'intention à la réalisation [Having a second child in Italy: From intention to realisation]. Population 66(2):434-450. https://doi.org/10.3917/popu.1102.0434.

Sharlin, Allan. 1986. Urban-rural differences in fertility in Europe during the demographic transition. In The decline of fertility in Europe, Hrsg. Ansley J. Coale, Susan C. Watkins, 234-260. Princeton: Princeton University Press.

Simmel, Georg. 1995. Die Großstädte und das Geistesleben. In Georg Simmel: Aufsätze und Abhandlungen 1901-1908, Bd. 1, Hrsg. Rüdiger Kramme, Angela Rammstedt, und Otthein Rammstedt, 116-131. Frankfurt am Main: Suhrkamp. 
Sobotka, Tomás, und Éva Beaujouan. 2014. Two Is best? The persistence of a two-child family ideal in Europe. Population and Development Review 40(3):391-419. https://doi.org/10.1111/j.1728-4457. 2014.00691.x.

Sobotka, Tomás, und Krystof Zeman. 2015. Fertility and partnership. In Religions in Vienna in the past, present and future: Key findings from the WIREL project, Hrsg. Anne Goujon, 8-9. Vienna: Vienna Institute of Demography.

Spéder, Zsolt, und Balázs Kapitány. 2009. How are time-dependent childbearing intentions realized? Realization, postponement, abandonment, bringing forward. European Journal of Population 25(4):503-523. https://doi.org/10.1007/s10680-009-9189-7.

Spéder, Zsolt, und Balázs Kapitány. 2014. Failure to realize fertility intentions: a key aspect of the postcommunist fertility transition. Population Reseach and Policy Review 33(3):393-418. https://doi.org/ 10.1007/s11113-013-9313-6.

Statistik Austria. 2015. Gliederung Österreichs in NUTS-Einheiten. Wien: Statistik Austria.

Statistik Austria. 2018. Wohnen 2017. Zahlen, Daten und Indikatoren der Wohnstatistik. Wien: Statistik Austria.

Statistik Austria. 2019a. Abgestimmte Erwerbsstatistik und Arbeitsstättenzählung. Wien: Statistik Austria.

Statistik Austria. 2019b. Betreuungsquoten. Wien: Statistik Austria.

Tönnies, Ferdinand. 1887. Gemeinschaft und Gesellschaft. Abhandlung des Communismus und des Socialismus als empirischer Culturformen. http://www.deutschestextarchiv.de/toennies_gemeinschaft_ 1887/9. Zugegriffen: 13. November 2020.

Trovato, Frank, und Carl F. Grindstaff. 1980. Decomposing the urban-rural fertility differential: Canada 1971. Rural Sociology 45(3):448-468.

Vignoli, Daniele, Francesca Rinesi, und Eleonora Mussino. 2013. A home to plan the first child? Fertility intentions and housing conditions in Italy. Population, Space and Place 19:60-71. https://doi.org/10. 1002/psp. 1716.

Vikat, Andres, Zsolt Spéder, Gijs Beets, Francesco Billari, Christoph Bühler, Aline Desesquelles, Tineke Fokkema, Jan M. Hoem, Alphonse MacDonald, Gerda Neyer, Ariane Pailhé, Antonella Pinnelli, und Anne Solaz. 2007. Generations and Gender Survey (GGS): Towards a better understanding of relationships and processes in the life course. Demographic Research 17(14):389-440. https://doi.org/10. 4054/DemRes.2007.17.14.

Wisbauer, Alexander, und Johannes Klotz. 2019. Demographische Strukturen und Trends nach städtischen und ländlichen Raumtypen. Statistische Nachrichten 2019(2):108-119.

Zeman, Kryštof, Tomáš Sobotka, Richard Gisser, und Maria Winkler-Dworak. 2019. Geburtenbarometer. Beobachtung der Fertilität in Österreich. Vienna Institute of Demography. http://www.birthbarometer. at/de/. Zugegriffen: 9. Okt. 2019.

Bernhard Riederer Mag. Dr., wissenschaftlicher Mitarbeiter am Institut für Soziologie der Universität Wien und am Institut für Demographie der Österreichischen Akademie der Wissenschaften. Forschungsinteressen: Familie/Elternschaft, soziale Ungleichheit/Lebensqualität, internationale und regionale Vergleiche.

Isabella Buber-Ennser DI Mag. Dr., Leiterin der Forschungsgruppe „Demographie Österreichs“ am Institut für Demographie der Österreichischen Akademie der Wissenschaften. Forschungsinteressen: Fertilität, Familiengründung, intergenerationale Transfers, Fluchtforschung. 